\title{
Applying Participatory Methods to Address Motivational Aspects in Informal Workplace Learning
}

\author{
doi:10.3991/ijac.v4i1.1412 \\ T. Holocher, B. Kieslinger and C. M. Fabian \\ Centre for Social Innovation/Technology \& Knowledge, Vienna, Austria
}

\begin{abstract}
Motivational aspects are core to successful knowledge sharing and collaborative learning experiences. However, it still remains one of the great challenges to overcome motivational barriers when it comes to introducing information systems for collaborative learning at the workplace. In the context of an international research project we have taken motivational aspects into account during the design phase and started a participatory process involving researchers, end-users, managers, designers and developers. As initial findings show, a continuous dialogue with endusers may contribute to creating a sense of ownership amongst them and become a motivational driver for the future use of the system.
\end{abstract}

Index Terms-collaborative learning, motivation, organizational learning, participatory design.

\section{INTRODUCTION}

Involving individuals at workplace in collaborative learning and knowledge sharing activities is still a major challenge for educational experts. A lot of research has been done on motivational aspects in organizational learning that focus on intrinsic and extrinsic motivations for learning and how these can be affected by external factors like organizational reward, management influence or social relationships. However, organizational research shows that a large majority of the intents to implement organizational learning and knowledge management processes and systems fail. In an international research project that aims to enhance collaborative learning and knowledge building activities across individual organizations we have chosen a participatory approach to involve different stakeholders during the whole research project and to immerse the researchers into the organizational context. Our hypothesis is that by making the design of the extended learning environment a joint effort between users, designers and researchers we create a sense of ownership and thus decrease motivational barriers for knowledge sharing and collaborative learning.

To test this hypothesis we are studying a business case which is embedded in a large multinational enterprise in the automotive sector. Together with a small business unit of this enterprise we intend to design and implement new learning services based on Web 2.0 principles that support cross-organizational collaborative workplace learning. The current Wiki system, which had been implemented mainly for knowledge sharing purposes, has shown little success so far. Thus, we will investigate the motivations and barriers for collaborative learning and develop con- cepts that not only include new technological services, but also consider the department's learning culture and working context. Discovering motivational drivers and overcoming barriers of collaborative learning and technology acceptance will be key to success.

The following section will discuss motivational theories in workplace learning and outline some of the most important motivational drivers and barriers. In section 3 we will elaborate on our participatory design model. Section 4 describes the specific business case and the following section outlines how the participatory design approach has been implemented in the specific business case. Finally section 6 will discuss the current findings followed by our conclusions.

\section{Motivational Aspects IN WorkPlace LeARNing}

Motivation theories for organizational learning basically distinguish between intrinsic and extrinsic motivation of knowledge workers. The former represents a mechanism where the ideal incentive system is given by the work content itself, which must be self-determined, satisfactory and fulfilling for the individual. Intrinsic motivation is valued for "its own sake and appears to be selfsustained" $[1$, p. 100]. It will flourish in circumstances, where the individuals' pursuit of autonomy, competence and relatedness is satisfied, and provides a "secure relational base" [2, p 71].

Extrinsic motivation focuses on indirect needs satisfaction, where an activity is undertaken in order to receive some separable outcome, like monetary compensation, awards, career opportunities, job security etc. Ryan and Deci [2] differentiate between 4 types of extrinsic motivation depending on the degree of the individual feeling of autonomy. The continuum begins with the least autonomous extrinsically motivated behavior, which is referred to as "externally regulated" and is driven by the need to satisfy an external demand or the potential benefit from external rewards. On the other end of the scale they define the most autonomous extrinsically motivated behavior, which is referred to as "integrated regulation". In this case the required behavior has been evaluated by the individual and brought into full accordance with their own values and needs.

When comparing individuals whose motivation is authentic (and thus intrinsic) with those who are externally controlled, the former show more interest, excitement, persistence and creativity than the latter, even when they show the same level of perceived competence and selfefficacy for a specific activity [2]. Thus motivational the- 
ory for learning stresses the importance of intrinsic motivation mechanisms, especially if tacit knowledge has to be shared sustainably [3].

According to Ryan and Deci [2] external factors can facilitate the internalization of extrinsic motivation. Again, the main factors to be supported are relatedness, namely in the sense of a feeling of belonging and being connected with others, perceived competence and perceived autonomy of learners.

Motivational research has also shown that extrinsic and intrinsic motivation mechanisms are not necessarily additive but can show more complex interactions between them. Extrinsic motivation can have an important impact on intrinsic motivation and vice versa, which is referred to as "crowding effects" [4]. The interplay of extrinsic and intrinsic motivation is strongly influenced by the organizational environment. Organizational culture, working autonomy, social norms, social relationships, legitimated hierarchies etc. are all factors that may impact the extent to which individuals are ready to actively, as well as sustainably, share and seek knowledge and to overcome learning barriers. Likewise, empirical research in the field of organizational learning has revealed several factors that strongly influence the collaborative knowledge sharing behavior of individuals in organizations. In the following we will elaborate the most important motivational drivers and barriers for collaborative learning and knowledge sharing processes that have been influencing our participatory design approach.

\section{A. Motivational drivers and barriers}

\section{1) Intrinsic motivational drivers}

\section{a) Enjoyment in helping others}

The act of contributing knowledge enhances the intrinsic satisfaction of an individual if helping others is considered as an activity that is for its own sake perceived as joyful and pleasurable. Connecting contributor and receiver of shared knowledge artifacts can actively enhance this enjoyment. When receivers post inquiries or questions, show interest in the sharer's knowledge, give recognition and feedback, the contributors prove to be more motivated to actively contribute knowledge artifacts to the collaborative learning system [5].

\section{b) Knowledge growth and increased performance}

Humans have the inherent tendency to seek for challenges, to extend and exercise their capacities, to explore and to learn. If learning is conducted for the pleasure of the activity itself, the individual is intrinsically motivated. If learning is driven by the individual's believe that seeking for knowledge results in new learning and personal access to new knowledge or innovations we may no longer speak of purely intrinsic motivation [6]. However, this learning behavior seems to accomplish the most internalized extrinsic motivation.

\section{2) Extrinsic motivational drivers}

\section{a) Reputation}

The fact that people believe that their professional reputation will increase within a group of relevant others (e.g. peers, management) if they contribute knowledge, is an important motivator for individuals to share knowledge in collaborative learning systems [6]. Externalizing one's knowledge, reflecting about current practices and docu- menting these via knowledge artifacts may thus be perceived as a means to document one's efforts and success and thereby to prove one's competences within a group of relevant others.

\section{b) Reciprocity}

Individuals who are motivated by reciprocity expect that in the future they will be able to benefit from their set of current contributions and the value created by their involvement in knowledge sharing. Such return-in-kind attitude mainly influences knowledge contribution behavior [6]: Extrinsic behaviour like reciprocity has proven to increase knowledge contribution in the early phase of adoption of new learning systems, but the influence weakens over time. In the post-adoption phase, intrinsic motivators like enjoyment in helping others, begin to stabilize and become the dominant beliefs. Schulz brings evidence that receiving knowledge from others stimulates a reciprocal flow of knowledge in the direction of the sender both horizontally and vertically [7]. Kim [8] analyzed that an increasing number of knowledge contributors encourages reciprocators to share more knowledge. Motivating reciprocity allows individuals to envisage that sharing knowledge will pay off in the future, even if the exact amount of benefit is not clear.

\section{c) Organizational reward}

Organizational reward is a very common means of external motivation. However, an external intervention from the management has always two aspects: the informing aspect and the control aspect. While the informing aspect exerts a positive influence on the perceived competence and strengthens the feeling of self-determinedness, the control aspect strengthens the perceived external control and the feeling of decreased autonomy. Therefore external interventions may have a negative or positive influence on intrinsic motivation, depending on whether the effects on constraints or on preferences dominate [3].

Various studies reflect on the diverse influence organizational reward has on the successful implementation and usage of collaborative learning environments. Wei He and Kwok-Kee Wei claim that economic reward is irrelevant to an individual's learning continuance behavior [6]. A meta-analysis on several empirical studies from Deci, Koestner and Ryan [9] provides additional evidence that tangible rewards even undermine intrinsic motivation for interesting tasks. This is particularly true for monetary compensations that are often perceived to be a controlling instrument. The authors thus stress the importance of other types of rewards that increase the professional competence and autonomy of individuals.

3) Factors influencing the work environment for knowledge sharing and seeking

\section{a) Culture of the work environment}

Organizational culture is another important external motivator. Social agreements, shared values, and beliefs are prerequisites for successful knowledge transfer [10]. In an organizational culture that involves norms of cooperation and norms of helping each other, knowledge transfer amongst employees is more probable than in hierarchical cultures in which colleagues compete with each other to win their superior's favor. Organizational culture influences assumptions about the importance of certain knowledge, it controls the relationships between the different levels of knowledge (organizational, group, individual), 
and most importantly it creates the context for social interaction [11]. In addition, it also determines the distribution of knowledge between organizations and individuals. Wilkesmann [10] suggests that the cultural aspects of involvement and consistency are most important in an environment that favors knowledge transfer. Involvement leads to the feeling of being important and being influential and therefore fosters the individuals' perception that their knowledge is relevant for others. Consistency within the corporate culture means that superiors practice what they preach and all employees commit to a set of shared core values and to a clear agreement on how to handle knowledge matters within the organization. A "knowledge-friendly" culture is one of the key success factors for collaborative learning but also the one that is the most difficult to establish. [12].

\section{b) Management influence}

The commitment of individuals to knowledge sharing and collaborative learning processes is strongly influenced by the perceived expectations of the management. The more an employee believes that information sharing is a correct and a socially expected type of workplace behavior the more will he or she contribute to or seek for knowledge [13]. Active support from the management in such activities via resources, time, recognition, a clear vision and guidelines can positively influence the knowledge seeking and contribution behavior of individuals [12]. In addition, results from empirical analysis show that employees' motivation to engage in learning and knowledge sharing activities increases if the management gives a good example and if individuals are involved in the formulation of the activities' objectives.

\section{c) Social relationship}

In any social process relationships matter. A lack of social relationship between the knowledge seeker and the knowledge contributors has been identified as an import barrier for knowledge sharing [11, 14]. When talking about social relationships the aspect of trust is particularly important. Individuals make judgments about the trustworthiness of their peers when it comes to sharing knowledge. Trust leads to the assurance that others will not ridicule or criticize the contributors' content, nor make abuse of the contributor's knowledge for individual purposes. Therefore the relationship between the parties and their history is an important foundation of trust. The behavior of individuals in the learning process is highly influenced by experiences of the past, which makes them anticipate the outcome of future learning activities $[15,16]$.

Trust is also an important factor when seeking for information. Whenever individuals decide to learn from externalized knowledge provided by others they run the risk to internalize content that might be misleading or not performance enhancing. Therefore individuals have to decide whether to trust in the quality of the provided externalized knowledge or not. The more complex and uncertain the external environment the more trust resides in the quality of the relationship rather than in the quality of the message. Trusting the other makes one more open to accept the knowledge offered by this person, therefore the trustworthiness is key to success in collaborative learning processes. The role of trust is seen as central by scholars such as Andrews. He claims that without trust, regardless of any formal knowledge-sharing requirement in place, employees will not share knowledge [15].

\section{d) Facilitating conditions}

Facilitating conditions matter in knowledge seeking behavior and are especially important for knowledge contribution behaviors. Training on how to use the system, facilitators that provide support during the whole process, and sufficient resources, especially time, are key factors in supporting learning activities. They provide individuals with the feeling of being competent enough to handle a specific learning activity. There are various means to help individuals to find orientation and take first steps to become knowledge contributors, such as the communication of transparent expectations and rules on the required learning outcome, as well as advertising examples of successful problem solving. The increased communication about "why" and "what for" they should share their knowledge helps to foster interest amongst employees and to understand the real benefits of the collaborative learning processes. Recognizing clear benefits is one of the most important motivators for participation [14].

\section{e) Effort}

Finally, the amount of cognitive energy contributed to the seeking or sharing of knowledge in terms of time and effort has to be minimized to successfully engage users in collaborative learning processes. Examples that have a negative influence on the adoption are the lack of appropriate tools, difficulty of searching and finding, and insufficiency of personalization [6]. Effort tends to be a less significant factor for the continuous usage of new learning systems, while the effect of social relationships and facilitating conditions become more important [13].

\section{PARTICIPATORY DESIGN}

As previously stated, our hypothesis in this work is that by making the design of the extended learning environment a joint effort between users, designers and researchers we create a sense of ownership and thus decrease motivational barriers for learning. We have chosen a participatory design approach where we involve the individual actors of our learning scenarios in the learning landscape design process.

The focus of the participatory design method is not on the explicit knowledge of employees but on the tacit aspects of human activity that are examined through design partnerships between researchers and participants. The role of the researcher is to make the tacit knowledge explicit, providing stakeholders with a more accurate understanding of how processes and systems work and how they can be improved. According to Kensing and MunkMadsen [17] researchers have to apply tools and techniques that not only elicit current working practices but also collect concrete experiences of the participants with the future system.

Participatory design is largely influenced by the organizational context and requires considerable flexibility in the selection and adaption of tools and technologies for knowledge creation. In addition, participatory research has faced criticism for not providing an explicit methodology for other scholars to follow.

To cope with this problem we elaborated the design conversion model - a framework that helps practitioners and researchers to structure their design activities around the four SECI-phases of the organizational knowledge creation theory from Nonaka and Takeuchi [18]. We will 
exemplify the taken approach in the context of an international research project and present some of the main findings of first user interactions.

\section{A. IntelLEO Design Conversion Model}

As described by Kieslinger et al [19] the four phases of Nonaka and Takeuchi's SECI model, namely socialization, externalization, combination and internalization are all addressed in the design conversion model (see Fig.1) and are executed in a joint effort between researchers, end-users, educational experts, system developers and managers. By covering the different stages in the design process we establish an iterative process of contextual investigation, idea generation, prototyping and evaluation.

During the socialization phase little intervention is done from the research perspective. Information exchange takes place mainly in an informal way and in face-to-face meetings of some of the participants.

The externalization phase is very important from the researcher's perspective. Here a lot of valuable data collection may be done. Together with the different stakeholders, interviews and focus groups are conducted. Depending on the stage of the technological development walkthrough and think aloud protocols may be performed, as well as learning diaries and the collection of questionnaires. Once a full prototype is up and running this phase will give valuable insights via monitoring and logging data.

In a third phase the collected knowledge needs to be combined, analyzed, transferred and generalized to a certain extent. Debriefing sessions and additional group discussions are planned for this stage as well as collaborative development of scenarios, storyboards, technical specifications, etc.

During the fourth phase, the internalization of processes and experiences, it is important to jointly reflect on phase two and three and feed all the results and insights back to the original contributors. Weblogs, summaries, presentations, storyboards and project reports are useful instruments during this phase.

The important innovation in this approach, which is a continuous and iterative process, is the fact that the employees/users are involved in the different stages and are thus designers of their learning activities, the involved processes and the technological support system. The researchers and developers play an important role in this interaction as they provide some norms and guidelines to the different stakeholders. They are mediators of a conversational translation from tacit knowledge to explicit context knowledge and further on to some more formalized use case descriptions, requirements definition and system design. The cyclic movement between an individualistic view and a collective view during the design, development and evaluation phase shapes the implementation framework as well as the design process itself.

\section{The Business CASE}

The business case that we are currently studying is located in a large multinational enterprise in the automotive sector. Within this organization a small business unit wants to implement an innovative approach to collaborative learning and knowledge building supported by collaborating learning services. The process should be implemented within the small unit, across various units in

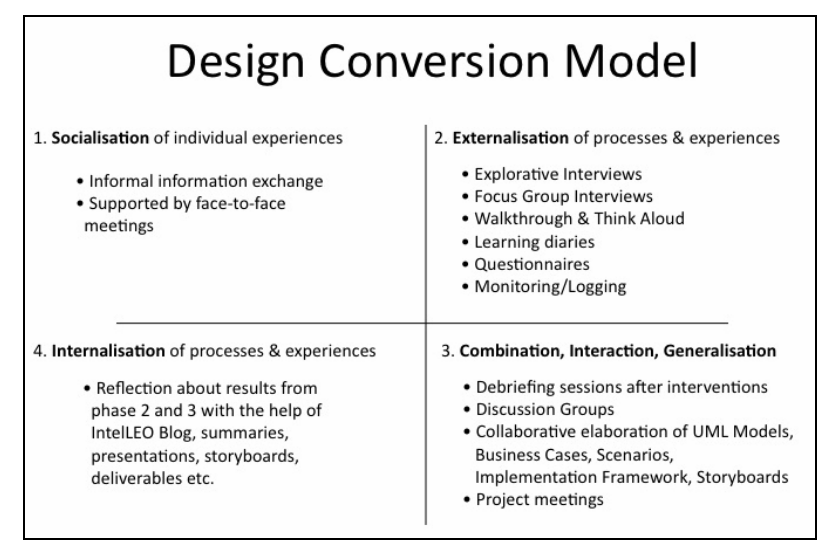

Figure 1. Design Conversion Model

the organization and extended to specific external cooperation partners.

Different instances have been defined covering core activities of workers in this unit that shall be supported by the new system and the corresponding andragogical model. These business case instances cover aspects such as the introduction of a newcomer to the internal working processes of the unit or a problem-based learning situation caused by unexpected changes in the work assignment of an experienced worker.

The main challenge for the implementation of such an innovation process is related to motivational aspects. The current Wiki system, which has been set up for the purpose of knowledge sharing, is not widely used. Since the staff members of the specific business unit are under continuous time pressure and work in a highly competitive situation they are reluctant to share knowledge and to document or externalize their expertise. Thus top management support for such organizational change processes is of high relevance.

\section{IMPLEMENTATION}

Our participatory design approach involves two groups of end-users. The first group consists of employees who are part of the core research project team and will be referred to as "facilitators" in this document. The second group is composed of employees who are not part of the core project team and are involved selectively in the design process. We will refer to them as "end-users".

The first phase of requirement elicitation started with an initial description of the business case by the facilitators, which was followed by explorative interviews with a group of end-uses covering existing working processes, current learning activities, the personal career development and organizational objectives. In addition employees came up with first ideas on how to improve current working practices and collaborative learning.

In a second step the individual interviews were summarized, analyzed in terms of commonalities and differences, and presented to the interviewees during a focus group. Together the participants reflected on the outcomes of the interviews, extended this knowledge base with more detailed information, and investigated more deeply on barriers and motivational drivers to use the collaborative Wiki for knowledge sharing. The reference to the collaborative learning system in use helped to better explain what hindered employees to actually share and seek for knowledge. 
To establish a continuous discussion flow with endusers a blog was set up that is regularly updated with the outcomes from the participatory design interventions. In addition the results of the first interviews were presented to the middle management in order to discuss identified areas of actions that could be pursued by the management. The involved employees and their direct superiors assisted during this presentation. Thus the employees' contribution during the participatory design process was given high visibility in front of the management, which in return additionally motivated the employees to stay involved.

While the work with end-users still focused on the contextual inquiry about current knowledge sharing practices, the facilitators were taken a step further. They were introduced to the andragogical concept of the project and developed first scenarios on how to implement the concept in their business case. In addition, facilitators, designers and technical engineers assessed first paper prototypes of future learning services in the framework of a pluralistic walkthrough.

As a next step, the outcome of this workshop will be integrated in the next version of paper prototypes which will then be presented to a group of end-users. In addition, a larger community of end-users will be invited to participate in the assessment of results from the initial interviews and from the focus group.

\section{RESULTS}

\section{A. Challenges and barriers}

In this section we will present the main results from the first interviews and the focus group discussions with endusers, summarizing the working challenges and motivational aspects, as well as barriers and drivers of collaborative knowledge sharing.

Employees of the investigated business unit face important challenges that derive from the intensive collaboration processes with other business units and external companies. The involved employees act as an interface between different operating departments and thus have to cope with complex business processes that contain manifold dependencies between diversified operational departments and complex information structures. Their role within this process is to collect decentralized information from the involved units, bundle and process this information and feed it into the further development process. Thus, these employees have to continually adapt their work to dynamic environmental changes of the sector and cope with high time pressure.

Complex access hierarchies and strong security regulations govern the information exchange between business units and external partners but contravene the requirement to quickly share information within a broad range of involved employees. Since there is no institutional education program for the type of job undertaken by the involved employees the most important resource for expert knowledge are colleagues and the knowledge pool generated by related projects. In the course of time the increasing number of successfully realized projects augments the own expert's knowledge. Gaining inter-divisional experience during their daily work helps employees to understand the diversified requirements of the involved business units and to find consent between the involved parties.
According to the interviews colleagues show a great willingness to share their knowledge but lack of time often constrains them. Moreover knowledge exchange is currently based on informal face-to-face meetings and thus not documented or externalized in order to become accessible to a broader range of employees. To address this problem a Wiki was introduced to the department with initial euphoria but the involvement of employees decreased quickly. The huge workload and the fact that they have to move pending tasks to the less busy working periods, leave hardly any time for the documentation and exchange of collected working experiences in a more formalized way. The dynamic work environment requires the continual extension of knowledge and practices, where employees can only partly rely on best practice from older projects. As employees have to manipulate a huge amount of written documents to successfully contribute to their projects, they do not feel motivated to produce even more written documents to exchange knowledge with colleagues. Especially, the participation on the Wiki misses a clear assignment as well as recognition from the management. Missing time to get to know the new software as well as difficulties with the usability of the Wiki have been identified as additional barriers.

\section{B. Motivational aspects}

Having derived some important issues regarding the current situation from the first interventions in the specific business case we started to specifically look into motivational aspects to overcome those challenges and barriers.

In general employees feel very motivated by the demanding role they play within the business process. They are proud of being able to successfully cope with their daily challenges, bearing responsibility and their personal degree of influence on the final outcomes of their work. Working closely together with different operational departments, having to understand and govern the diversified needs, and finding concerted solutions for all involved parties, is a demanding but highly esteemed job. The interviewees appreciate the inherent need to continually learn and enhance their competences, which is required by the dynamic environment of the sector. In addition, the recognition of their efforts and competences by the management is an important extrinsic motivator that was mentioned by employees. Overall, the feeling of autonomy is rather strong within this group of employees.

The potential benefits that the employees expect from a collaborative learning system are basically an accelerated information exchange between the operating units and external companies, a more transparent and documented exchange of learning experiences between colleagues and thus a quicker access to relevant knowledge.

As a first step of the implementation process end-users required the commitment of the management, not only in stating a good example but also in paying attention to the employees' efforts in the knowledge sharing process and granting resources to support it. The learning environment should create visibility for the employees' efforts within the group of peers but even more important at the management level.

Interviewees requested that the documentation and sharing of experiences became part of the department's culture and was integrated into current working processes that dominate their daily routine. Activities on the virtual 
platform should be connected with encounters in the real world, where the electronically shared documents are the basis for learning that takes place when talking about this information.

The employees ask for assistance for the generation of the initial content base, not only by a facilitator who takes over responsibility for the learning environment and pushes employees to use the platform but also by some expert who could support his colleagues in taking the initial hurdle of formulating and documenting collected experiences. As an alternative means of documentation and knowledge sharing the participants suggested considering videos in order to decrease the burden of writing and reading.

As employees have to handle a large number of software services already, the new learning environment should either access information from existing repositories or existing services should move to the new platform. In any case, a duplication of content and effort through parallel running systems should be avoided.

During the focus group discussion employees came up with first format ideas and an initial content pool. Especially in the adoption phase first positive experiences with the new learning environment and a grasp of the potential benefit of the new services are considered important. To their mind an initial content base would also allow a flow of reciprocal information for those who add content. Ideally, an increasing group of active contributors would then motivate less active knowledge sharers to get involved.

Group dynamics play an important role when motivating end-users to become active content producers. In addition social relationships have been perceived as motivational drivers, when they foster the contact between colleges and their exchange of learning experiences. To allow a maximum number of employees to participate in the new learning environment without contradicting strict security regulations, the flexible and easy definition of access rights to externalized content and learning groups will be key to success.

To increase the perceived competence of the employees for handling the new learning environment, interviewees favor a playful approach that helps to get accustomed with the new services and that is not necessarily related to the real business of the company but addresses more intrinsically motivated areas of activity.

\section{CONCLUSIONS}

This paper discusses some theoretical concepts, a methodological approach and interim findings of an ongoing research project that focuses on cross-organizational workplace learning. In the design phase researchers started a collaborative discussion process with a set of stakeholders in a concrete organizational context. In the specific business case from the automotive industry employees are selectively liberated from their daily duties to reflect about current learning practices and how to improve them. The researchers' role is to enable employees to make their tacit knowledge about current working processes explicit and share it with their colleagues, as well as designers and developers of the future learning system. The first interventions revealed important aspects on current working processes, as well as motivational drivers and barriers for collaborative workplace learning. It helped to better understand the context and culture of in- volved employees and their initial requirements for collaborative learning.

Being still at an early stage of investigation one of the main contributions of this first phase was to gain awareness and interest for the project among involved employees, their colleagues and their management. The expectations from the employees' side are high and their ideas and requirements have to be carefully considered during the next conceptual phase. Next steps will involve a larger group of employees to test first results from the explorative investigations. With the help of paper prototypes we will try to translate initial user-requirements into new services that will be assessed by end-users.

As previously stated, in this project we are still in the middle of implementing the participatory design approach. So far we have gained very important input from the involved users that helped to shape the first prototype even if only in paper form. We realized the importance of motivational aspects and that a participatory design can be of value here. It is very important to adapt the methodology to the context and to be very sensitive about when to involve whom. As some critics of participatory design have rightly pointed out [20] motivation might also decrease if people are involved too much or at the wrong stage of the design process.

\section{ACKNOWLEDGMENT}

IntelLEO (http://www.intelleo.org) "Intelligent Learning Extended Organization" is a collaborative research project. We would like to thank the whole research team of IntelLEO. This work is supported in part by the European Union under Grant FP7-ICT-231590.

\section{REFERENCES}

[1] Deci, E. L. "Intrinsic Motivation." Plenum Press, New York, 1975.

[2] Ryan, R. M., Deci, E. L. "Self-determination theory and the facilitation of intrinsic motivation, social development, and wellbeing." American Psychologist 55, 2000, p. 68-78. doi:10.1037/0003-066X.55.1.68

[3] Osterloh, M., Frey, B. "Motivation, Knowledge Transfer, and Organizational Forms." Organization Science 11(5), 2000, p. 538. doi:10.1287/orsc.11.5.538.15204

[4] Frey, B. S., Jegen, R. "Motivation Crowding Theory." Journal of Economic Surveys 15(5), 2001, p. 589 - 611. doi:10.1111/1467$\underline{6419.00150}$

[5] Liechtenstein, S., Hunter, A. "Toward a Receiver-Based Theory of Knowledge Sharing." Current Issues in Knowledge Management, 2008, p. 86-100.

[6] He, W., Wei, K.-K. "What drives continued knowledge sharing? An investigation of knowledge-contribution and -seeking beliefs." Decision Support Systems 46, 2009, p. 826-838. doi:10.1016/j.dss.2008.11.007

[7] Schulz, M. "The uncertain relevance of newness: organizational learning and knowledge flows." Academy of Management Journal 44(4), 2001, p. 661-681. doi:10.2307/3069409

[8] Kim, J., Sang, M.L., Olson, D.L. "Human Effect of Knowledge Sharing: Cooperative Type and Reciprocity Level in Community of Practice." Current Issues in Knowledge Management, 2008, p. 66-85.

[9] Deci, E. L., Koestner, R., Ryan, R.M. „A meta-analytic review of experiments examining the effects of extrinsic rewards on intrinsic motivation" Psychological Bulletin. Vol 125(6), Nov 1999, 627668. doi:10.1037/0033-2909.125.6.627

[10] Wilkesmann, U., Wilkesmann, M., Virgillito, A. "The Absence of Cooperation Is Not Necessarily Defection: Structural and Motivational contraints of Knowledge Transfer in a Social Dilemma 
Situation." Organization Studies 30(10), 2009, p. 1141-1164. doi: $10.1177 / 0170840609344385$

[11] Ipe, M. "Knowledge Sharing in Organisations: A conceptual framework", Human Resource Development Review, Dec 2003, Vol 2, No 4, pp 337. doi:10.1177/1534484303257985

[12] Davenport, T. H., De Long, D.W., Beers, M.C. "Successful Knowledge Management Projects." The Knowledge Management Yearbook, 1999-2000.

[13] Venkatesh, Viswanath; Morris, Michael G.; Davis, Gordon B.; Davis, Fred D., "User acceptance of information technology: Toward a unified view", MIS Quarterly, 2003, 27, 3, 425-478.

[14] Ardichvili, A., Page, W., Wentling, T. "Motivation and barriers to participation in virtual knowledge-sharing communities of practice", Journal of Knowledge Management, 2003, 7, 1. doi: $10.1108 / 13673270310463626$

[15] Andrews, K., Delahaye, B. "Influences on Knowledge Processes in Organizational Learning: The Psychological Filter." Journal of Management Studies 37(6), 2000. doi:10.1111/1467-6486.00204

[16] Nonaka, I., Konno, N. "The Concept of "Ba": Building a Foundation for Knowledge Creation". California Management Review 40(3), 1998, p. 40-54.
[17] Kensing, F., Munk-Madsen, A. "Participatory Design: Structure in the Toolbox." Communication of the ACM 36(4), 1993,p. 78 doi:10.1145/153571.163278

[18] Nonaka, I., \& Takeuchi, H. "The knowledge-creating company: How Japanese companies create the dynamics of innovation". Oxford: Oxford University Press, 1995.

[19] Kieslinger, B. P., K.; Fabian, C.M. "A Participatory Design Approach for the Support of Collaborative Learning and Knowledge Building in Networked Organizations." International Journal for Advanced Corporate Learning 2(3), 2009.

[20] Wagner E.L., Piccoli, G. "Moving Beyond User Participation to Achieve Successful IS Design". Communications of the ACM, December 20007. Vol.50, No.12.

\section{AUTHORS}

T. Holocher, B. Kieslinger and C. M. Fabian are with the Centre for Social Innovation/Technology \& Knowledge, Vienna, Austria.

Submitted July $29^{\text {th }}, 2010$. Published as resubmitted by the authors January $27^{\text {th }}, 2011$. 